\title{
A Linear Regression Framework for the Receiver Operating Characteristic (ROC) Curve Analysis
}

\section{Zheng Zhang ${ }^{1 *}$ and Ying Huang ${ }^{2}$}

${ }^{1}$ Department of Biostatistics and Center for Statistical Sciences, Brown University, Providence, RI 02912, USA

${ }^{2}$ Fred Hutchinson Cancer Research Center, Seattle, WA 98109, USA

\begin{abstract}
The receiver operating characteristic (ROC) curve has been a popular statistical tool for characterizing the discriminating power of a classifier, such as a biomarker or an imaging modality for disease screening or diagnosis. It has been recognized that the accuracy of a given procedure may depend on some underlying factors, such as subject's demographic characteristics or disease risk factors, among others. Non-parametric- or parametric-based methods tend to be either inefficient or cumbersome when evaluating effect of multiple covariates is the main focus. Here we propose a semi-parametric linear regression framework to model covariate effect. It allows the estimation of sensitivity at given specificity to vary according to the covariates and provides a way to model the area under the ROC curve indirectly. Estimation procedure and asymptotic theory are presented. Extensive simulation studies have been conducted to investigate the validity of the proposed method. We illustrate the new method on a diagnostic test dataset.
\end{abstract}

Keywords: AUC; Covariate effect; Linear regression; ROC curve; Sensitivity

\section{Introduction}

New advances in medical technology have produced an array of potentially powerful tools to screen for and diagnose various medical conditions. Effective screening and accurate diagnosis can ensure optimal treatment and improve prognosis. Before a screening or diagnostic test can be applied in a clinical setting, rigorous statistical assessment of its performance in discriminating the diseased state from the non-diseased state is required. For tests measured on the continuous scale, the receiver operating characteristic (ROC) curve is a common statistical tool for describing the performance of such tests [1]. Let $D$ be disease status ( 1 for disease and 0 otherwise) and $Y$ the test result with positivity defined whenever $Y \geq c$. Define the true positive fraction $(\mathrm{TPF}(\mathrm{c}))$ and the false positive fraction $(\mathrm{FPF}(\mathrm{c}))$ as $P[Y \geq c \mid D$ $=1]$ and $P[Y \geq c \mid D=0]$, where TPF and 1- FPF are also called test's sensitivity and specificity. The ROC curve is a plot of $T P F(c)$ versus $F P F(c)$ when the threshold $c$ ranges from $-\infty$ to $\infty$. Alternatively, the ROC curve can be written as a function of $t$, by defining $S_{D}(c)=P[Y \geq c$ $\mid D=1]=T P F(c), S_{\bar{D}}(c)=P[Y \geq c \mid D=0]=F P F(c)$, where $D$ and $\bar{D}$ indicate the diseased and non-diseased population, then

$$
R O C(t)=S_{D}\left(S_{\bar{D}}^{-1}(t)\right), t \in(0,1)
$$

Different approaches have been proposed to estimate an ROC curve. Nonparametrically, we can obtain the empirical ROC curve based on $\widehat{T P F}(c)=\sum_{i=1}^{n_{D}} I\left[Y_{D_{i}} \geq c\right] / n_{D}$ and $\widehat{F P F}(c)=\sum_{j=1}^{n_{\bar{D}}} I\left[Y_{\bar{D}_{j}} \geq c\right] / n_{\bar{D}}$, where $n_{D}$ and $n_{\bar{D}}$ are number of diseased and non-diseased observations respectively.

Alternatively, we can estimate the ROC curve parametrically by assuming a distributional form for $S_{D}$ and $S_{\bar{D}}$ and then calculating the induced ROC curve with equation (1). The derived ROC curve, however, is not invariant to transformation of the test results. Pepe [2] notes that ROC curve describes only the relationship between the distributions of $S_{D}$ and $S_{\bar{D}}$, not the distributions themselves. Semiparametric estimators that directly model the ROC curve as a parametric function without specifying the underlying distributions of $S_{D}$ and $S_{\bar{D}}$ provides a desirable alternative.
Semiparametric estimations of the ROC curve starts by first specifying a parametric model of the ROC curve, where the binormal model is most popular [3], and is defined as the following:

$$
R O C(t)=\Phi\left(\alpha_{0}+\alpha_{1} \Phi^{-1}(t)\right),
$$

with $\Phi$ being the cdf of the standard normal distribution. We call $\alpha_{0}$ the intercept and $\alpha_{1}$ the slope of the binormal ROC curve. The binormal ROC curve was originally derived from normally distributed test results, where $Y_{D} \sim N\left(\mu_{D}, \sigma_{D}^{2}\right)$ and $Y_{\bar{D}} \sim N\left(\mu_{\bar{D}}, \sigma_{\bar{D}}^{2}\right)$, the resulting ROC curve has $\alpha_{0}=\left(\mu_{D}-\mu_{\bar{D}}\right) / \sigma_{D}$ and $\alpha_{1}=\sigma_{\bar{D}} / \sigma_{D}$. However, since the ROC curve is invariant to strictly increasing transformations of $Y$, to say that the ROC curve is binormal simply means that there exists some strictly increasing transformation, which would simultaneously transform the raw data, $Y_{\bar{D}}$ and $Y_{D}$, into normally distributed random variables. In addition, under the binormal model, the area under the ROC curve (AUC) can be written as $A U C=\Phi\left(\alpha_{0} / \sqrt{1+\alpha_{1}^{2}}\right)$.

Various semiparametric methods have been proposed to estimate the ROC curve under the binormal assumption. LABROC [4] is a maximum likelihood-based procedure for ordinal test results. Specifically, it categorizes continuous data and then applies the Dorfman and Alf algorithm [5] to the categorized data. ROC-GLM [6$8]$ is a binary regression based method. Pepe and Cai [9] and Cai [10] estimated the ROC curve using the concept of placement values (PV).

The development of our method is motivated by noting that the binormal model in equation (2) essentially states that $\Phi^{-1}(T P F)$ and

*Corresponding author: Zheng Zhang, Department of Biostatistics and Center for Statistical Sciences, Brown University, 121 South Main Street Providence, RI 02912, USA, Tel: 401-863-2578; E-mail: zzhang@stat.brown.edu

Received December 06, 2011; Accepted March 22, 2012; Published March 23 , 2012

Citation: Zhang Z, Huang Y (2012) A Linear Regression Framework for the Receiver Operating Characteristic (ROC) Curve Analysis. J Biomet Biostat 3:137. doi:10.4172/2155-6180.1000137

Copyright: $\odot 2012$ Zhang Z, et al. This is an open-access article distributed unde the terms of the Creative Commons Attribution License, which permits unrestricted use, distribution, and reproduction in any medium, provided the original author and source are credited. 
$\Phi^{-1}(F P F)$ have a linear relationship. Hence we propose to fit a linear line through the points of $\left(\Phi^{-1}(\widehat{F P F}), \Phi^{-1}(\widehat{T P F})\right)$ and obtain estimates of $\alpha_{0}$ and $\alpha_{1}$ by the least squares technique, and consequently, estimate the ROC curve itself. This method is intuitive, conceptually easy to understand, and very easy to implement. More importantly, it can be readily extended to allow for covariate $(Z)$ effects on the ROC curve by writing

$$
\Phi^{-1}\left(R O C_{Z}(t)\right)=\alpha_{0}+\alpha_{1} \Phi^{-1}(t)+\theta Z
$$

Our linear regression approach provides a simple and widely accessible algorithm for fitting such models.

Hsieh and Turnbull [11] described a weighted least squares approach to estimate the binormal ROC curve. For continuous data, their approach groups the data into a pre-determined number (independent of the number of observations) of categories, and the largest they chose is 12 . Our framework is similar in spirits but allows significant improvement in efficiency by eliminating the need of grouping, as will be shown later in the next section. Moreover, our framework offers the flexibility to model additional covariate effects and/or to model a segment of the ROC curve. In many applications only a part of the ROC curve is of interest [12]. Restricting fitting of model $(3)$ to a subrange $[a, b]$ within $(0,1)$ is likely to confer robustness over the region of interest compared with fitting over the entire $(0,1)$ range.

\section{Linear Regression Framework and Estimation}

\section{Estimation}

Write the empirical ROC curve as $\widehat{R O C}_{e}(t)=\hat{S}_{D}\left(\hat{S}_{\bar{D}}^{-1}(t)\right)$, where $\hat{S}_{\bar{D}}^{-1}(t)=\inf \left\{y: \widehat{S_{\bar{D}}}(y) \leq t\right\}$. As shown in Appendix I,

$$
\sqrt{n_{\bar{D}}}\left[\Phi^{-1}\left(\widehat{R O C_{e}(.)}\right)-\Phi^{-1}(R O C(.))\right] \stackrel{D}{\rightarrow} N(0, \Sigma),
$$

meaning that the process converges to a mean zero Gaussian process with variance-covariance function

$$
\begin{aligned}
& \Sigma(s, t)=1 /\left\{\phi\left(\alpha_{0}+\alpha_{1} \Phi^{-1}(s)\right) \phi\left(\alpha_{0}+\alpha_{1} \Phi^{-1}(t)\right)\right\}\left[\lambda \Sigma_{1}(s, t)+\Sigma_{2}(s, t)\right], \\
& \text { where } \Sigma_{1}(s, t)=R O C(t) \wedge R O C(s)-R O C(s) R O C(t), \\
& \Sigma_{2}(s, t)=\alpha_{1}^{2} \phi\left(\alpha_{0}+\alpha_{1} \Phi^{-1}(s)\right) \phi\left(\alpha_{0}+\alpha_{1} \Phi^{-1}(t)\right) \\
& /\left\{\phi\left(\Phi^{-1}(t)\right) \phi\left(\Phi^{-1}(s)\right)\right\}(t \wedge s-t s),
\end{aligned}
$$

$0<s<1,0<t<1$, and $\lambda$ is the limit of the ratio $n_{\bar{D}} / n_{D}$ as $n_{\bar{D}}$ approaches infinity.

\section{We propose the following estimating procedure:}

1. For a fixed boundary point $(a, b)$, choose the set $T=\left\{t_{p}\right\}$ such that $0<a<t_{1}<\ldots<t_{p}<\ldots<b<1$. For each $t_{p}$, find the smallest threshold value $c_{p}$, such that $t_{p} \geq \widehat{F P F}\left(c_{p}\right)=\sum_{j=1}^{n_{\bar{D}}} I\left[Y_{\bar{D}_{j}} \geq c_{p}\right] / n_{\bar{D}}$;

2. Calculate $\widehat{R O C}\left(t_{p}\right)=\widehat{T P F}\left(c_{p}\right)=\sum_{i=1}^{n_{D}} I\left[Y_{D_{i}} \geq c_{p}\right] / n_{D}$, exclude $\left(t_{p}, \widehat{R O C}\left(t_{p}\right)\right)$ if $\widehat{R O C}\left(t_{p}\right)$ is either 0 or 1 ;

3. Set up the linear regression model as:

$$
\Phi^{-1}\left(\widehat{R O C}\left(t_{p}\right)\right)=\alpha_{0}+\alpha_{1} \Phi^{-1}\left(t_{p}\right)+\varepsilon_{p},
$$

where the normalized error vector, $\sqrt{n_{\bar{D}}} \varepsilon$, is distributed as multivariate normal with mean 0 and asymptotic covariance matrix $\Sigma$;

4. Define the design matrix $M$ as

$$
\begin{aligned}
& M^{\prime}=\left(\begin{array}{cccc}
1 & \cdots & 1 & \cdots \\
\Phi^{-1}\left(t_{1}\right) & \cdots & \Phi^{-1}\left(t_{1}\right) & \ldots
\end{array}\right) \text { and the vector } \\
& \Phi^{-1}(\widehat{\operatorname{ROC}}(t))^{\prime}=\left(\Phi^{-1}\left(\widehat{\operatorname{ROC}}\left(t_{1}\right)\right), \ldots, \Phi^{-1}\left(\widehat{\operatorname{ROC}}\left(t_{p}\right)\right), \ldots\right) .
\end{aligned}
$$

5. Calculate the ordinary least squares(OLS) estimator of $\alpha$ as

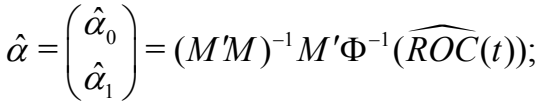

6. Then the estimated ROC curve and its associated AUC are

$$
\begin{aligned}
& \widehat{R O C}(t)=\Phi\left(\hat{\alpha}_{0}+\hat{\alpha}_{1} \Phi^{-1}(t)\right) \text { and } \\
& \widehat{A U C}=\Phi\left(\hat{\alpha}_{0} / \sqrt{1+\hat{\alpha}_{1}^{2}}\right)
\end{aligned}
$$

\section{Asymptotic theory}

We develop the asymptotic distribution of the OLS estimator $\hat{\alpha}=\left(\hat{\alpha}_{0}, \hat{\alpha}_{1}\right)$ under the following assumptions: $\left\{Y_{D_{i}}\right\}$ and $\left\{Y_{\bar{D}_{j}}\right\}$ are i.i.d. random variables with a survival functions $S_{D}$ and $S_{\bar{D}}$ and density functions $f_{D}$ and $f_{\bar{D}}$, respectively; $n_{\bar{D}} / n_{D} \rightarrow \lambda, 0<\lambda<\infty$, as $n_{\bar{D}} \rightarrow \infty$; the slope of the ROC curve, $f_{D}\left(S_{\bar{D}}^{-1}(t)\right) / f_{\bar{D}}\left(S_{\bar{D}}^{-1}(t)\right)$, is bounded on the subinterval $[a, b]$ of $(0,1), 0<\mathrm{a}<\mathrm{b}<1$.

\section{Theorem 1}

where

$$
\sqrt{n_{D}}\left(\begin{array}{l}
\hat{\alpha}_{0}-\alpha_{0} \\
\hat{\alpha}_{1}-\alpha_{1}
\end{array}\right) \stackrel{D}{\rightarrow} N\left(\left(\begin{array}{l}
0 \\
0
\end{array}\right), A \Sigma_{A} A^{T}\right),
$$

$$
A=\left[\int_{a}^{b}\left(\begin{array}{cc}
1 & \Phi^{-1}(t) \\
\Phi^{-1}(t) & \left(\Phi^{-1}(t)\right)^{2}
\end{array}\right) d(t)\right]^{-1},
$$

and

$$
\Sigma_{A}=\left(\begin{array}{cc}
\sigma_{A_{1}}^{2} & \sigma_{A_{12}} \\
\sigma_{A_{12}} & \sigma_{A_{2}}^{2}
\end{array}\right),
$$

where

$$
\begin{gathered}
\sigma_{A_{1}}^{2}=\lambda \int_{a}^{b} \int_{a}^{b} J_{1}(s) J_{1}(t)(R O C(s) \wedge R O C(t)-R O C(s) R O C(t)) d s d t \\
+\alpha_{1}^{2} \int_{a}^{b} \int_{a}^{b} J_{2}(s) J_{2}(t)(s \wedge t-s t) d s d t, \\
\sigma_{A_{2}}^{2}=\lambda \int_{a}^{b} \int_{a}^{b} K_{1}(s) K_{1}(t)(R O C(s) \wedge R O C(t)-R O C(s) R O C(t)) d s d t \\
+\alpha_{1}^{2} \int_{a}^{b} \int_{a}^{b} K_{2}(s) K_{2}(t)(s \wedge t-s t) d s d t, \\
\sigma_{A_{12}}=\lambda \int_{a}^{b} \int_{a}^{b} K_{1}(s) J_{1}(t)(R O C(s) \wedge R O C(t)-R O C(s) R O C(t)) d s d t \\
+\alpha_{1}^{2} \int_{a}^{b} \int_{a}^{b} J_{2}(s) K_{2}(t)(s \wedge t-s t) d s d t,
\end{gathered}
$$

with $J_{1}(s)=\left(\varphi\left(\Phi^{-1}(R O C(s))\right)\right)^{-1}, J_{2}(s)=\left(\varphi\left(\Phi^{-1}(s)\right)\right)^{-1}, K_{1}(s)=\Phi^{-1}(s) J_{1}(s)$ and $K_{2}(s)=\Phi^{-1}(s) J_{2}(s)$.

Proof: The proof for Theorem 1 can be found in the Appendix II.

\section{Asymptotic efficiency relative to $\mathrm{HT}$ method}

Here we compare the asymptotic efficiency between the OLS estimator and the estimator derived using Hsieh \& Turnbull's method (HT) for estimating $\alpha_{0}, \alpha_{1}$, and $R O C(t)$ at $t=0.2,0.4,0.7$, when $\lambda$ (the 
limit of the ratio of $n_{\bar{D}}$ and $\left.n_{D}\right)$ varies from 0.5 to 2 . We choose $\left(\alpha_{0}, \alpha_{1}\right)$ $=(1.2,0.45)$ so that the area under the ROC curve (AUC) is 0.863 and the ROC curve takes values of $0.794,0.861$ and 0.924 at $t=0.2,0.4,0.7$, respectively. Note that for $h(t)=\left(\begin{array}{c}1 \\ \Phi^{-1}(t)\end{array}\right)$, let $\Sigma$ denote the asymptotic variance of $\sqrt{n_{\bar{D}}}\left(\hat{\alpha}_{0}, \hat{\alpha}_{1}\right)$ from an ROC modeling method (OLS or HT), the asymptotic variance expression for the corresponding $\widehat{R O C}(t)$ are:

$$
\operatorname{var}(\widehat{R O C}(t)) \doteq 1 / n_{\bar{D}}\left\{\left(\phi\left(\alpha_{0}+\alpha_{1} \Phi^{-1}(t)\right)\right)^{2} h^{T}(t) \Sigma h(t)\right\}
$$

Table 1 shows the asymptotic efficiency of HT relative to OLS. For the OLS estimator, the boundary points $(a, b)$ chosen are $(0.0001$, 0.9999). For the HT estimator, the number of categories chosen is eight. Note that asymptotically, the OLS estimator can lead to substantial efficiency gain compared to the HT estimator. The biggest improvement is seen when estimating $\alpha_{1}$ with efficiency gain above $50 \%$. The efficiency gain in estimating points on the ROC curve varies from $3 \%$ to $20 \%$ depending on the point of interest (Table 1).

\section{Regression Model with Discrete Covariates}

Suppose there are $K$ categories that potentially could overlap with each other. For $k=1, \ldots, K$, let $Z(k)$ be a vector of length $K-1$, with value 1 for the $k-1^{\text {th }}$ element and zero else where (so Z(1) is a vector of zeros), and let $n_{\bar{D}_{k}}$ be the number of non-diseased observations in category $k$. Suppose the ROC curve within category $k$ is characterized by

$$
\Phi^{-1}\left(R O C_{\mathrm{k}}(t)\right)=\beta_{1}+\gamma \Phi^{-1}(t)+\theta^{T} Z(k)
$$

where $\theta=\left(\beta_{2}, \ldots, \beta_{k}\right)^{T}$.

That is, for subset 1 (the reference subset)

$$
\Phi^{-1}\left(R O C_{1}(t)\right)=\beta_{1}+\gamma \Phi^{-1}(t)
$$

and for subset $k, k=2, \ldots, K$,

$$
\Phi^{-1}\left(R O C_{\mathrm{k}}(t)\right)=\beta_{1}+\gamma \Phi^{-1}(t)+\beta_{k}
$$

Hence $\beta_{k}$ is the difference in the intercept parameter of the ROC curves between subset $k$ and the reference subset. The parameter of interest here is $\theta^{*}=\left(\beta_{1}, \ldots, \beta_{\mathrm{k}}, \gamma\right)^{T}$. The underlying assumptions for equation (5) is that there exists an unknown monotone increasing function $h_{1}$, such that $h_{1}\left(Y_{\bar{D}, 1}\right) \sim N(0,1)$ and $h_{1}\left(Y_{D, 1}\right): N\left(\beta_{1} / \gamma, 1 / \gamma^{2}\right)$. Similarly, for subsequent subset $k, k=2,3, \ldots, K,(6)$ implies there exists an unknown monotone increasing function $h_{\mathrm{k}}$, such that $h_{k}\left(Y_{\bar{D}, k}\right) \sim N(0,1)$ and $h_{1}\left(Y_{D, 1}\right) \sim N\left(\beta_{1} / \gamma, 1 / \gamma^{2}\right)$. Notice that $h_{\mathrm{k}}$ s are not required to be the same for different $k$.

Let $\widehat{R O C}_{k}(t)$ be the empirical estimate of $\operatorname{ROC}_{k}(t)$ based on data from category $k$. Like in the case of equation (4), $\sqrt{n_{\bar{D}, k}}\left(\Phi^{-1}\left(\widehat{R O C_{k}(t)}\right)-\Phi^{-1}\left(R O C_{k}(t)\right)\right)$ converge to a Gaussian process, therefore $\Phi^{-1} R O C_{k}(t)$ can be approximated by $\Phi^{-1}\left(\widehat{R O C}_{k}(t)\right)$, which motivates the following estimation procedure:

1. Calculate pairs of $\left(t_{p}, \widehat{\operatorname{ROC}}_{k}\left(t_{p}\right)\right)$ for each subset separately;

2. Let design matrix $D$ be

$$
D=\left(\begin{array}{ccccc}
M & O & O & . . & O \\
M & M_{1} & O & . . & O \\
M & O & M_{1} & . . & O \\
\cdot & \cdot & . & . . & \cdot \\
M & O & O & . . & M_{1}
\end{array}\right)
$$

where $M=\left(\begin{array}{ccc}1 & \cdots & 1 \\ \Phi^{-1}\left(t_{1}\right) & \cdots & \Phi^{-1}\left(t_{p}\right)\end{array}\right)^{T}, M_{1}$ is a vector of length $p$ with constant value of one, and $O$ is a zero value matrix.

3.Let $\tilde{Y}=\left(\tilde{Y}_{1}, \tilde{Y}_{2}, \ldots, \tilde{Y}_{K}\right)^{T}$ and $\tilde{Y}_{k}=\left(\Phi^{-1}\left(\widehat{R O C}_{k}\left(t_{1}\right)\right) \ldots \Phi^{-1}\left(\widehat{R O C}_{k}\left(t_{p}\right)\right)\right)$;

4. Our linear model is: for the reference subset,

$\Phi^{-1}\left(\widehat{R O C}_{1}\left(t_{p}\right)\right) \doteq \beta_{1}+\gamma_{1} \Phi^{-1}\left(t_{p}\right)+\varepsilon_{1}$,

where $\sqrt{n_{\bar{D}, 1}} \varepsilon_{1}$ is normally distributed with mean 0 and asymptotic covariance matrix $\Sigma_{r, 1 ;}$ and for subset $k, k=2,3, . ., K$

$$
\Phi^{-1}\left(\widehat{R O C}_{k}\left(t_{p}\right)\right) \doteq \beta_{1}+\gamma_{1} \Phi^{-1}\left(t_{p}\right)+\beta_{k}+\varepsilon_{k},
$$

where $\sqrt{n_{\bar{D}, k}} \varepsilon_{k}$ is normally distributed with mean 0 and asymptotic covariance matrix $\Sigma_{r, k}$;

\section{Our OLS estimator for $\theta^{*}$ is \\ $\hat{\theta}^{*}=\left(D^{\prime} D\right)^{-1} D^{\prime} \tilde{Y}$}

The above method assumes covariate effects can be explained adequately by the difference in the intercept parameter $\left(\alpha_{0}\right)$. If in addition we allow the slope of a binormal ROC curve to be different across covariate categories by assuming

$$
\Phi^{-1}\left(R O C_{k}(t)\right)=\beta_{1}+\gamma_{1} \Phi^{-1}(t)+\left(1 \Phi^{-1}(\mathrm{t})\right) \theta^{T} Z(k),
$$

where

$$
\theta=\left(\begin{array}{lll}
\beta_{2} & \cdots & \beta_{k} \\
\gamma_{2} & \cdots & \gamma_{k}
\end{array}\right)^{T}
$$

then the parameter of interest is $\theta^{*}=\left(\beta_{1}, \gamma_{1}, \ldots, \beta_{k}, \gamma_{k}\right)^{T}$. The estimating procedure is similar to the case where only intercept parameters vary, but with $M_{1}$ replaced by $M$ in (7). Inference for the significance of $\theta^{*}$ in both settings can be achieved by estimating the variance of $\theta^{*}$ with the bootstrap resampling method.

\section{Simulation}

\section{Estimation of the ROC curve}

The estimation procedure specified in the previous section starts with the choice of the false positive set $T$. Although in theory, any

\begin{tabular}{|c|c|c|c|c|}
\hline$\lambda$ & $\alpha_{0}$ & $\alpha_{1}$ & $R(0.2)$ & $R(0.4)$ \\
\hline 0.5 & 1.10 & 1.72 & 1.09 & 1.06 \\
\hline 1 & 1.10 & 1.64 & 1.05 & 1.20 \\
\hline 2 & 1.10 & 1.59 & 1.03 & 1.20 \\
\hline
\end{tabular}

Table 1: Asymptotic efficiency of OLS relative to HT (with 8 categories). 
chosen set of $T$ would yield estimators with the same asymptotic property, their small sample properties need to be investigated. An obvious starting point is to choose the collection of the observed false positive fractions that fall into the interval $[a, b]$, we call this observed FP (OFP) method. In the case where there are no ties in the test results of non-diseased subjects, this is equivalent to the method selecting the subset of $\left\{1 / n_{\bar{D}}, 2 / n_{\bar{D}}, \ldots . .,\left(n_{\bar{D}}-1\right) / n_{\bar{D}}\right\}$ within $[a, b]$, which we call the equal fraction (EF) method. Another possible choice is to divide interval $[a, b]$ into $n_{\bar{D}}-1$ equally spaced sub-intervals and choose the midpoints of those subintervals to be the set $T$ (midpoint(MP) method). For the last two methods, the number of points in $T$ always equal to $n_{\bar{D}}-1$ regardless of the length of $[a, b]$.

We compare the performance of the OLS estimators between those three different methods of selecting $T$ (OFP, EF and MP). We simulate dataset both with and without ties and vary the values of $[a, b]$ to estimate either a full curve or a partial ROC curve.

First, we generate $Y_{\bar{D}} \sim N(0,1)$ and $Y_{D} \sim N\left(\alpha_{0} / \alpha_{1}, 1 / \alpha_{1}^{2}\right)$ . The resulting ROC curve follows a binormal model: $R O C(t)=\Phi\left(\alpha_{0}\right.$ $\left.+\alpha_{1} \Phi^{-1}(t)\right)$. The parameters $\left(\alpha_{0}, \alpha_{1}\right)$ are chosen to be $(1.2,0.45)$, corresponding to an area under the curve (AUC) value of 0.863 and a partial AUC (pAUC) value of 0.142 for $t \in(0,0.2)$. With sample size of $n_{D}=n_{\bar{D}}=100$, we compare the biases and sampling standard errors in estimating $\mathrm{AUC}, \operatorname{ROC}(0.2), \operatorname{ROC}(0.4)$ and $\mathrm{ROC}(0.7)$ in the full curve estimation and $\mathrm{pAUC}(0.2)$ and $\mathrm{ROC}(0.1)$ in the partial curve estimation. To simulate data with ties, we chose $20 \%$ tied values within each population.

From Table 2, when the test results have no ties, midpoint(MP) method has the best performance. When data has ties, observed false positive fraction(OFP) method has the smallest bias when estimating the entire curve, but MP has the best performance for estimating the partial curve. We recommend using either method MP or OFP in practice and we choose MP method for the subsequent simulations (Table 2).

Next we compare performance of the OLS estimator with two existing semiparametric ROC modeling approach (ROC-GLM and $\mathrm{PV})$, which have been shown to have good performance among others. Table 3 summarizes relative biases and standard errors for the three estimators for estimating either a full or a partial ROC curve. We observe that when the full ROC curve is of interest, the OLS and the GLM estimators have comparable performances while the PV estimator has somewhat larger biases. When estimating a partial ROC curve, however, the OLS estimator can have substantially smaller bias compared to other estimators (Table 3).

In Table 4, we demonstrate performance of the OLS estimator relative to the nonparametric ROC estimator and the parametric ROC estimator assuming normal case and control distributions after Box-Cox transformation. We generate data as $Y_{\bar{D}} \sim \operatorname{LOGN}(0,1)$ and $Y_{D} \sim \operatorname{LOGN}\left(\alpha_{0} / \alpha_{1}, 1 / \alpha_{1}^{2}\right)$ so the resulting ROC curve is the same as above. We further modify the data as following: (i) inflating all data points by two fold (scaled log normal distribution); (ii) increasing all data points by 2 units (shifted log normal distribution); (iii) $\exp \left(Y^{1 / 3}\right)$ (non Box-Cox). For the parametric method, Box-Cox transformation is applied to $Y_{\bar{D}}$ and $Y_{D}$ separately before the fitting of a normal distribution. For log normal data or scaled log normal data, the OLS estimator and the parametric estimator combined with Box-Cox transformation have similar performances. For shifted log normal and non Box-Cox data, however, the parametric estimator performs poorly with a large bias even after a Box-Cox transformation. Both the OLS estimator and the nonparametric estimator are unbiased in these scenarios with the former substantially more efficient (Table 4).

Lastly, we perform simulation studies to investigate the use of large sample inference for ROC curve and AUC based on the OLS estimator. Table 5 shows the mean estimated asymptotic error and the coverage of corresponding $95 \%$ Wald confidence intervals. The variance estimate from the asymptotic theory reflects the actual sampling variance and the coverage of $95 \%$ confidence intervals is excellent (Table 5).

\section{Application to DPOAE Data Set}

The DPOAE data set was first published by Stover et al. [13].

\begin{tabular}{|c|c|c|c|c|}
\hline Parameter & True value & OFP & EF & MP \\
\hline \multicolumn{5}{|l|}{ Percent of ties: $0 \%$} \\
\hline \multicolumn{5}{|l|}{$(a, b)=(0.0001,0.9999)$} \\
\hline$R(0.2)$ & 0.794 & $0.791(0.039)$ & $0.792(0.039)$ & $0.795(0.038)$ \\
\hline$R(0.4)$ & 0.861 & $0.860(0.031)$ & $0.860(0.031)$ & $0.862(0.031)$ \\
\hline$R(0.7)$ & 0.924 & $0.923(0.024)$ & $0.923(0.024)$ & $0.924(0.024)$ \\
\hline AUC & 0.863 & $0.861(0.027)$ & $0.861(0.027)$ & $0.863(0.027)$ \\
\hline \multicolumn{5}{|l|}{$(a, b)=(0.0001,0.2)$} \\
\hline$R(0.1)$ & 0.733 & $0.727(0.049)$ & $0.729(0.048)$ & $0.733(0.048)$ \\
\hline $\mathrm{pAUC}(0.2)$ & 0.142 & $0.141(0.010)$ & $0.141(0.010)$ & $0.142(0.010)$ \\
\hline \multicolumn{5}{|l|}{ Percent of ties:20\% } \\
\hline \multicolumn{5}{|l|}{$(a, b)=(0.0001,0.9999)$} \\
\hline $\mathrm{R}(0.2)$ & 0.794 & $0.794(0.043)$ & $0.805(0.041)$ & $0.798(0.042)$ \\
\hline $\mathrm{R}(0.4)$ & 0.861 & $0.862(0.034)$ & $0.879(0.031)$ & $0.864(0.034)$ \\
\hline $\mathrm{R}(0.7)$ & 0.924 & $0.924(0.026)$ & $0.942(0.022)$ & $0.926(0.025)$ \\
\hline AUC & 0.863 & $0.863(0.030)$ & $0.875(0.027)$ & $0.865(0.030)$ \\
\hline \multicolumn{5}{|l|}{$(a, b)=(0.0001,0.2)$} \\
\hline $\mathrm{R}(0.1)$ & 0.733 & $0.728(0.053)$ & $0.739(0.051)$ & $0.734(0.053)$ \\
\hline pAUC(0.2) & 0.142 & $0.141(0.011)$ & $0.143(0.010)$ & $0.143(0.011)$ \\
\hline
\end{tabular}

Table 2: Inference of the ROC curve by the semiparametric least squares based method (OLS) with different choices of the false positive sets (OFP: observed false positive fraction, EF: equal fraction or MP: midpoint method). Cases and controls are drawn from normal distributions. $\left(\alpha_{0}, \alpha_{1}\right)=(1.2,0.45)$. $n_{D}, n_{\bar{D}}=(100,100)$. Sample means and sampling standard errors from 1000 simulations are shown. 
Citation: Zhang Z, Huang Y (2012) A Linear Regression Framework for the Receiver Operating Characteristic (ROC) Curve Analysis. J Biomet Biostat 3:137. doi:10.4172/2155-6180.1000137

Page 5 of 7

\begin{tabular}{|c|c|c|c|}
\hline Parameter & True value & OLS & GLM \\
\hline$(a, b)=(0.0001,0.9999)$ & & & $1.09(0.150)$ \\
\hline$\alpha_{0}$ & 1.2 & $2.42(0.083)$ & $1.46(0.152)$ \\
\hline$\alpha_{1}$ & 0.45 & $-0.14(0.040)$ & $0.16(0.038)$ \\
\hline$R(0.2)$ & 0.794 & $-0.02(0.031)$ & $0.12(0.031)$ \\
\hline$R(0.4)$ & 0.861 & $-0.02(0.023)$ & $-0.002(0.023)$ \\
\hline$R(0.7)$ & 0.924 & $0.15(0.032)$ & $0.38(0.024)$ \\
\hline$(a, b)=(0.0001,0.2)$ & 1.2 & $0.18(0.246)$ & $-1.79(0.235)$ \\
\hline$\alpha_{0}$ & 0.45 & $-0.62(0.141)$ & $-6.82(0.130)$ \\
\hline$R(0.05)$ & 0.677 & $0.11(0.051)$ & $1.27(0.049)$ \\
\hline$R(0.1)$ & 0.733 & $-0.02(0.046)$ & $14.045(0.266)$ \\
\hline
\end{tabular}

Table 3: Inference of the ROC curve by the semiparametric least squares based method (OLS), the ROC-GLM method (GLM) and the placement value method (PV). Cases and controls are drawn from normal distributions. $\left(\alpha_{0}, \alpha_{1}\right)=(1.2,0.45),\left(n_{D}, n_{\bar{D}}\right)=(100,100)$. Relative bias and sampling standard error from 500 simulations are shown. Relative bias $=$ bias/true value $\times 100 \%$.

\begin{tabular}{|c|c|c|c|c|c|}
\hline Parameter & True value & Distribution & OLS & EMP & RES \\
\hline $\mathrm{R}(0.2)$ & 0.794 & lognormal & $0.796(0.039)$ & $0.795(0.044)$ & $0.795(0.036)$ \\
\hline $\mathrm{R}(0.4)$ & 0.861 & & $0.863(0.031)$ & $0.864(0.036)$ & $0.862(0.030)$ \\
\hline $\mathrm{R}(0.7)$ & 0.924 & & $0.926(0.023)$ & $0.926(0.027)$ & $0.924(0.023)$ \\
\hline$A \cup C$ & 0.863 & & $0.864(0.027)$ & $0.864(0.027)$ & $0.863(0.026)$ \\
\hline $\mathrm{R}(0.2)$ & 0.794 & lognormal-scaled & $0.795(0.039)$ & $0.796(0.043)$ & $0.794(0.036)$ \\
\hline $\mathrm{R}(0.4)$ & 0.861 & & $0.862(0.032)$ & $0.863(0.037)$ & $0.861(0.030)$ \\
\hline $\mathrm{R}(0.7)$ & 0.924 & & $0.925(0.024)$ & $0.925(0.028)$ & $0.923(0.023)$ \\
\hline AUC & 0.863 & & $0.863(0.028)$ & $0.863(0.028)$ & $0.862(0.026)$ \\
\hline $\mathrm{R}(0.2)$ & 0.794 & lognormal-shift & $0.794(0.039)$ & $0.795(0.043)$ & $0.818(0.043)$ \\
\hline $\mathrm{R}(0.4)$ & 0.861 & & $0.861(0.031)$ & $0.861(0.036)$ & $0.900(0.031)$ \\
\hline $\mathrm{R}(0.7)$ & 0.924 & & $0.923(0.023)$ & $0.923(0.027)$ & $0.961(0.017)$ \\
\hline AUC & 0.863 & & $0.862(0.027)$ & $0.862(0.027)$ & $0.888(0.027)$ \\
\hline $\mathrm{R}(0.2)$ & 0.794 & non Box-Cox & $0.796(0.038)$ & $0.796(0.044)$ & $0.764(0.051)$ \\
\hline $\mathrm{R}(0.4)$ & 0.861 & & $0.863(0.031)$ & $0.864(0.036)$ & $0.887(0.037)$ \\
\hline $\mathrm{R}(0.7)$ & 0.924 & & $0.926(0.023)$ & $0.926(0.027)$ & $0.968(0.017)$ \\
\hline AUC & 0.863 & & $0.864(0.027)$ & $0.864(0.027)$ & $0.862(0.029)$ \\
\hline
\end{tabular}

Table 4: Inference of the ROC curve by the semiparametric least squares based method (OLS), the nonparametric method (EMP) and the parametric method (RES). Cases and controls are drawn from log-normal distributions and modified as noted. $\left(\alpha_{0}, \alpha_{1}\right)=(1.2,0.45)$. $\left(n_{D}, n_{\bar{D}}\right)=(100,100)$. Sample means and sampling standard errors from 1000 simulations are shown.

\begin{tabular}{|c|c|c|c|c|}
\hline & Relative Bias & SSE & ASE & $\mathrm{CP}$ \\
\hline & \multicolumn{4}{|c|}{$\left(n_{D}, n_{\bar{D}}\right)=(100,100)$} \\
\hline$\alpha_{0}$ & $2.4 \%$ & 0.163 & 0.151 & $94.8 \%$ \\
\hline$\alpha_{1}$ & $2.2 \%$ & 0.085 & 0.083 & $95.4 \%$ \\
\hline $\mathrm{R}(0.2)$ & $0.4 \%$ & 0.039 & 0.039 & $94.6 \%$ \\
\hline$R(0.4)$ & $0.3 \%$ & 0.032 & 0.031 & $93.4 \%$ \\
\hline \multirow[t]{2}{*}{$\mathrm{R}(0.7)$} & $0.1 \%$ & 0.025 & 0.023 & $90.8 \%$ \\
\hline & \multicolumn{4}{|c|}{$\left(n_{D}, n_{\bar{D}}\right)=(100,50)$} \\
\hline$\alpha_{0}$ & $0.9 \%$ & 0.159 & 0.157 & $95.0 \%$ \\
\hline$a_{1}$ & $2.9 \%$ & 0.088 & 0.088 & $94.6 \%$ \\
\hline $\mathrm{R}(0.2)$ & $-0.3 \%$ & 0.041 & 0.042 & $94.6 \%$ \\
\hline $\mathrm{R}(0.4)$ & $-0.1 \%$ & 0.033 & 0.033 & $94.6 \%$ \\
\hline \multirow[t]{2}{*}{$\mathrm{R}(0.7)$} & $-0.1 \%$ & 0.025 & 0.024 & $93.8 \%$ \\
\hline & \multicolumn{4}{|c|}{$\left(n_{D}, n_{\bar{D}}\right)=(50,100)$} \\
\hline$\alpha_{0}$ & $1.1 \%$ & 0.205 & 0.208 & $96.4 \%$ \\
\hline$\alpha_{1}$ & $0.9 \%$ & 0.101 & 0.113 & $97.2 \%$ \\
\hline $\mathrm{R}(0.2)$ & $-0.2 \%$ & 0.053 & 0.053 & $93.8 \%$ \\
\hline $\mathrm{R}(0.4)$ & $-0.2 \%$ & 0.041 & 0.043 & $94.4 \%$ \\
\hline $\mathrm{R}(0.7)$ & $-0.3 \%$ & 0.030 & 0.033 & $94.6 \%$ \\
\hline
\end{tabular}

Table 5: Result of 1000 simulations to evaluate the application of inference based on asymptotic theory to finite sample studies. $\left(\alpha_{0}, \alpha_{1}\right)=(1.2,0.45)$. Relative bias $=$ bias / true value x 100\%; SSE: sampling standard error; ASE: mean estimated standard error using asymptotic theory; CP: coverage of $95 \%$ Wald-confidence intervals using estimated asymptotic error. 


\begin{tabular}{|c|c|c|c|c|c|c|}
\hline \multicolumn{7}{|c|}{ (Frequency, intensity) } \\
\hline & Parameter & Estimate & Std.Err & $P$ value & AUC & $\operatorname{ROC}(0.1)$ \\
\hline$(1001,55)$ & $\beta_{1}$ & 1.73 & 0.26 & $<0.0001$ & & \\
\hline$(1001,55)$ & $\mathrm{V}_{1}$ & 0.82 & 0.12 & $<0.0001$ & 0.910 & 0.751 \\
\hline$(1001,60)$ & $\beta_{2}$ & -0.20 & 0.14 & 0.1455 & 0.882 & 0.684 \\
\hline$(1001,65)$ & $\beta_{3}$ & -0.36 & 0.19 & 0.0607 & 0.855 & 0.625 \\
\hline$(1416,55)$ & $\beta_{4}$ & 0.81 & 0.28 & $0.0041^{*}$ & 0.975 & 0.932 \\
\hline$(1416,60)$ & $\beta_{5}$ & 0.36 & 0.29 & 0.2172 & 0.947 & 0.851 \\
\hline$(1416,65)$ & $\beta_{6}$ & 0.19 & 0.28 & 0.5064 & 0.931 & 0.808 \\
\hline$(2002,55)$ & $\beta_{7}$ & 0.47 & 0.29 & 0.1052 & 0.956 & 0.875 \\
\hline$(2002,60)$ & $\beta_{8}$ & 0.42 & 0.28 & 0.1293 & 0.952 & 0.864 \\
\hline$(2002,65)$ & $\beta_{9}$ & 0.10 & 0.27 & 0.7019 & 0.921 & 0.782 \\
\hline
\end{tabular}

Table 6: Covariates effects on the ROC curves estimated by the the OLS method for the DPOAE test.

DPOAE stands for distortion product otoacoustic emission, which is an audiology test used to separate normal-hearing from hearingimpaired ears.

The test is administrated under nine different auditory stimulus conditions with three levels of frequency $(1001,1416$ and $2002 \mathrm{~Hz})$ and three levels of intensity (55, 60 and $65 \mathrm{~dB}$ SPL). A total of 210 subjects were included in the study. The subjects were considered cases with hearing impairment at a given frequency if their audiometric threshold exceeds 20dB HL measured by a behavior test (gold standard). Each subject was tested in only one ear. Test result is the negative signal-tonoise ratio, -SNR, with higher value being more indicative of hearing impairment. The objective of the analysis is to determine the optimal setting for the clinical use of DPOAE to separate normal from hearingimpaired ears, but bear in mind an ear may be determined to be hearing impaired or normal at different frequencies.

We partition the data into nine subsets, corresponding to the nine test settings. Data is analyzed by the method specified in (8) where both intercept and slope parameters vary across subsets. The set $T$ is chosen by the midpoint method from $(0.0001,0.9999)$. We choose the reference subset (subset 1 ) to be the test setting with frequency value of $1001 \mathrm{~Hz}$ and intensity value of $55 \mathrm{~dB}$ SPL. Let $\left(\beta_{1}, \gamma_{1}\right)$ be the intercept and slope estimates for the ROC curve for setting $(1001,55)$ and the subsequent $\beta_{k}$ and $\gamma_{k}$, with $k=2, \ldots, 9$, represent the differences in the intercept and slope parameters of the ROC curves between subset $k$ and subset 1 . None of the $\hat{\gamma}_{k}$ is statistically significantly different from 0 at $\alpha=0.05 \alpha=0.05$ level (data are not shown).

We also develop a $\chi^{2}$ test statistic $\hat{\gamma}^{\prime} \hat{\Sigma} \hat{\gamma}$, where $\gamma=\left(\gamma_{2}, \ldots \gamma_{9}\right)$ and $\hat{\Sigma}_{\gamma}$ is the estimated covariance matrix of $\hat{\gamma}$. Write the null hypothesis as $H_{0}: \gamma=0$ and compare the above statistic with a $\chi^{2}$ distribution with 8 degrees of freedom gives a P-value of 0.84 , suggesting insignificant slope terms consistent with the result from testing the significance of each $\gamma_{k}$ separately.

We re-analyze the data with the slope terms omitted and Table 6 summarizes the results. We can see that among the nine test settings, the setting $(1416,55)\left(\beta_{4}\right)$ has the largest intercept estimate and the difference from the intercept of the reference setting $(1001,55)$ is statistically significant $(\mathrm{p}=0.0041)$. The $\mathrm{p}$ values for other parameters $\left(\beta_{2} \beta_{3} \beta_{5}\right.$ to $\beta_{9)}$ are not significant. The estimated ROC curve for setting $(1416,55)$ is $R O C(t)=\Phi\left(2.54+0.82 \Phi^{-1}(\mathrm{t})\right)$ with estimated AUC value of 0.975 , which is the largest among all settings. The performance of the test declines with increasing intensity at each fixed frequency value. This analysis suggests $(1416 \mathrm{~Hz}, 55 \mathrm{~dB}$ SPL $)$ is a better test setting than the reference setting. It has also shown that at fixed false positive level of $10 \%$, the expected sensitivities for the nine test settings range from $62.5 \%$ to $93.2 \%$, demonstrating the importance of choosing an optimal test setting (Table 6).

\section{Concluding Remarks}

This manuscript proposes a semiparametric OLS method to estimate and compare performance of diagnostic tests and more generally, to assess potential covariate effects on the test performance. The asymptotic distribution theory for the OLS estimator is developed for the ROC curve estimation, in which the estimators are shown to be consistent and asymptotically normally distributed. For modeling covariate effects, we recommend boostrap resampling for variance estimation.

Our proposed estimator provides useful addition to the field of rank-based semiparametric ROC modeling. Those semiparametric approaches are more robust than parametric approaches, by assuming a functional form on the ROC curve itself but not the test results and thus invariant to monotone transformation of the test results. At the same time, they offer better efficiency compared to nonparametric method. We have done extensive simulations to compare the proposed OLS estimator with two other commonly used semiparametric ROC modeling methods (the ROC-GLM and placement value based method), and found the OLS estimator has comparable performance in general and slightly better performance in some scenarios [12]. The OLS estimator, however is much more intuitive compared to other estimators and very easy to implement using standard linear regression software, which could make it particularly appealing to clinical audience.

In summary, the proposed linear regression framework provides an unified approach for the ROC curve analysis. It can be used to estimate the ROC curve, as well as model covariate effect. The application of ROC curve goes beyond the medical diagnostic field and it can be used for evaluating any discrimination tools. It is, and will continue to be an important and exciting area to engage in research.

\section{Acknowledgements}

The authors would like to acknowledge Margaret S. Pepe for her invaluable contribution to the development of this manuscript. The work is partially funded by $\mathrm{NIH}$ grant GM54438 and P30CA015704. 
Citation: Zhang Z, Huang Y (2012) A Linear Regression Framework for the Receiver Operating Characteristic (ROC) Curve Analysis. J Biomet Biostat 3:137. doi:10.4172/2155-6180.1000137

Page 7 of 7

\section{References}

1. Swets JA, Pickett RM (1982) Evaluation of diagnostic systems: method from signal detection theory. Academic Press.

2. Pepe MS (2004) The statistical evaluation of medical tests for classification and prediction. Oxford University Press, United Kingdom.

3. Metz CE, Kronman HB (1980) Statistical significance tests for binormal roc curves. J Math Psychol 22: 218-243.

4. Metz CE, Herman BA, Shen JH (1998) Maximum likelihood estimation of receiver operating characteristic (ROC) curves from continuously-distributed data. Stat Med 17: 1033-1053.

5. Dorfman DD, Alf E (1969) Maximum likelihood estimation of parameters of signal detection theory and determination of confidence intervals-rating method data. J Math Psychol 6: 487-496.

6. Pepe MS (1997) A regression modelling framework for receiver operating characteristic curves in medical diagnostic testing. Biometrika 84: 595-608.

7. Pepe MS (2000) An interpretation for the ROC curve and inference using GLM procedures. Biometrics 56: 352-359.
8. Alonzo TA, Pepe MS (2002) Distribution-free ROC analysis using binary regression techniques. Biostatistics 3: 421-432.

9. Pepe MS, Cai T (2004) The analysis of placement values for evaluating discriminatory measures. Biometrics 60: 528-535.

10. Cai T (2004) Semi-parametric ROC regression analysis with placement values Biostatistics 5: 45-60.

11. Hsieh F, Turnbull BW (1996) Nonparametric and semiparametric estimation of the receiver operating characteristic curve. Ann Statist 24: 25-40.

12. Baker SG, Pinsky PF (2001) A proposed design and analysis for comparing digital and analog mammography. J Am Statist Assoc 96: 421-428.

13. Stover L, Gorga MP, Neely ST, Montoya D (1996) Toward optimizing the clinical utility of distortion product otoacoustic emission measurements. J Acoust Soc Am 100: 956-967.

14. Zhang Z (2004) Semiparametric least squares analysis of the receiver operating characteristic curve. University of Washington.
Submit your next manuscript and get advantages of OMICS Group submissions

Unique features:

User friendly/feasible website-translation of your paper to 50 world's leading languages

Audio Version of published paper

Digital articles to share and explore

Special features:

200 Open Access Journals

15,000 editorial team

21 days rapid review process

Quality and quick editorial, review and publication processing

- Indexing at PubMed (partial), Scopus, DOAJ, EBSCO, Index Copernicus and Google Scholar etc

Sharing Option: Social Networking Enabled

- Authors, Reviewers and Editors rewarded with online Scientific Credits

Better discount for your subsequent articles

Submit your manuscript at: http://www.omicsonline.org/submission 


\section{Appendix I: Proof of Equation (4)}

Hsieh and Turnbull(1996, Theorem 2.1) showed, as $n_{\bar{D}} \rightarrow \infty$,

$$
\sup _{0 \leq t \leq 1}\left|\widehat{R O C}_{e}(t)-R O C(t)\right| \rightarrow 0 \text { a.s. }
$$

They also showed (Theorem 2.2) for independent observations, under the above conditions, there exists a probability space on which one can define sequences of two independent versions of Brownian bridges $\left(B_{1}^{(n)}, B_{2}^{(n)}, 0 \leq t \leq 1\right)$, and the following statement holds:

$$
\sqrt{n_{\bar{D}}}\left(\widehat{R O C}_{e}(t)-R O C(t)\right)=\sqrt{\lambda} B_{1}^{(n)}(R O C(t))+\alpha_{1} \frac{\phi\left(\alpha_{0}+\alpha_{1} \Phi^{-1}(t)\right)}{\phi\left(\Phi^{-1}(t)\right)} B_{2}^{(n)}(t)+o\left(n^{-\frac{1}{2}}(\log n)^{2}\right)
$$

a.s. uniformly on $[a, b], 0<a<b<1$.

The above two theorems stated the strong consistency and strong approximation properties for the ROC curve.

Fix $t \in[a, b]$, by intermediate value theorem,

$$
\Phi^{-1}\left(\widehat{R O C}_{e}(t)\right)-\Phi^{-1}(R O C(t))=\phi\left(\Phi^{-1}\left(R O C^{*}(t)\right)\right)\left(\widehat{R O C}_{e}(t)-R O C(t)\right)
$$

where

$$
\left|R O C^{*}(t)-R O C(t)\right| \leq\left|\widehat{R O C}_{e}(t)-R O C(t)\right|
$$

Therefore, $\operatorname{ROC}^{*}(t) \rightarrow R O C(t)$ a.s. by (9)

From continuous mapping theorem:

$$
\phi\left(\Phi^{-1}\left(\operatorname{ROC}^{*}(t)\right)\right) \rightarrow \phi\left(\Phi^{-1}(R O C(t))\right) \text { a.s. }
$$

Notice

$$
\phi\left(\Phi^{-1}(R O C(t))\right)=\frac{1}{\phi\left(\Phi^{-1}(R O C(t))\right)}=\frac{1}{\phi\left(\alpha_{0}+\alpha_{1} \Phi^{-1}(t)\right)}
$$

Then we have

$$
\sqrt{n_{\bar{D}}}\left(\Phi^{-1}\left(\widehat{R O C}_{e}(t)\right)-\Phi^{-1}(R O C(t))\right) \rightarrow \frac{1}{\phi\left(\alpha_{0}+\alpha_{1} \Phi^{-1}(t)\right)} \sqrt{n_{\bar{D}}}\left(\widehat{R O C}_{e}(t)-R O C(t)\right)
$$




$$
=\sqrt{\lambda} \frac{1}{\phi\left(\Phi^{-1}(R O C(t))\right)} B_{1}^{(n)}(R O C(t))+\alpha_{1} \frac{1}{\phi\left(\Phi^{-1}(t)\right)} B_{2}^{(n)}(t)+o\left(n^{-\frac{1}{2}}(\log n)^{2}\right)
$$

a.s. uniformly on $[a, b], 0<a<b<1$.

Let

$$
V_{n}(t)=\sqrt{n_{\bar{D}}}\left(\Phi^{-1}\left(\widehat{R O C}_{e}(t)\right)-\Phi^{-1}(R O C(t))\right)
$$

$V(t)=\sqrt{\lambda} \frac{1}{\phi\left(\Phi^{-1}(R O C(t))\right)} B_{1}^{(n)}(R O C(t))+\alpha_{1} \frac{1}{\phi\left(\Phi^{-1}(t)\right)} B_{2}^{(n)}(t)$

Equation (16) implies $V_{n}(t) \Rightarrow V(t)$ in $\left(D[a, b],\|\cdot\|_{\infty}\right)$

Equation (4) resulted from the fact that $\mathrm{V}(\mathrm{t})$ is the sum of two independent Brownian Bridges.

\section{Appendix II: Proof of Theorems 1}

Theorem 1 is a direct consequence of the equation (4), namely, asymptotic normality of the empirical ROC estimates. 